\title{
ON SOME INVERSE PROBLEMS FOR A NONLINEAR TRANSPORT EQUATION
}

\author{
by
}

\author{
T. John Connolly and David J.N. Wall \\ Department of Mathematics \& Statistics \\ University of Canterbury \\ Christchurch, New Zealand
}

No. 145

August, 1996

Email address of D.J.N. Wall: D. Wall @math.canterbury.ac.nz

Shorttitle: Inverse problems for a Transport Equation

1995 Physics and Astronomy Classification Scheme: PACS: 02.30Jr, 03.40Kf 


\title{
ON SOME INVERSE PROBLEMS FOR A NONLINEAR TRANSPORT EQUATION
}

\author{
T. JOHN CONNOLLY AND DAVID J.N. WALL
}

\begin{abstract}
Inverse problems for nonlinear transport or one-way wave equation are considered. Analytic solutions to the inverse problems are given and it is shown, for some signalling problems, that the inverse problem of determination of a wavespeed functional is well-posed, and that the wavespeed can be uniquely determined. However, it is shown that the inverse problems of reconstruction of a source functional are ill-posed, although the source is still uniquely determined.
\end{abstract}

\section{INTRODUCTION}

Inverse problems for a linear one-way wave equation have recently been considered by [10]. In references [2] and [1], inverse problems associated with wave propagation problems involving parameters that have both spatial and temporal variation were examined. One of the motives for examination of these problems, was to develop techniques which would be useful in inverse problems associated with nonlinear wave equations, via quasi-linearisation methods.

The problems considered in this paper are to illustrate the type of behaviour that can be expected with inverse problems connected with nonlinear wave equations, and to show such problems can be effectively solved. We note here that an inverse problem for the eiconal equation from geometric optics, also a nonlinear first order hyperbolic equation, has previously been considered by Connolly [6].

A novel feature of the presentation within this paper is that analytic solutions are provided for the majority of the inverse problems presented.

We consider inverse signaling problems for the quasi-linear one-way wave equation in $\S 2$. A solution for the inverse problem of functional wavespeed reconstruction is given that is well-posed. It also is shown that the solution to this inverse problem is unique. Solutions of some inverse source problems, for a semi-linear one-way wave equation are presented in $\S 3$. These problems are shown to be ill-posed.

\section{Quasi-linear Transmission Problem}

Consider the source free, quasi-linear one-way wave equation

$$
u_{t}+c(u) u_{x}=0, \quad 0<x<\ell, \quad t>0,
$$

Date: 7 August 1996. 
for the dependent variable $u(x, t)$, as an initial-boundary value problem with the side conditions

$$
\begin{aligned}
u(0, t)=h(t), & 0 \leq t \leq \infty, \\
u(x, 0)=f(x), & 0 \leq x \leq \ell .
\end{aligned}
$$

Here, we assume the parameter $c$, is the wavespeed and is purely a function of the dependent variable. For convenience in the sequel we define the wave slowness

$$
\varsigma=1 / c
$$

At the boundary end point $x=\ell$, it is assumed that the field satisfies the appropriate consistency requirement, for the values propagated along the characteristics that cross this boundary line.

An implicit solution to equation (2.1) can be found to be

$$
u(x, t)=f(x-c(u) t) \mathrm{H}(x-t c(u))+h(t-\varsigma(u) x) \mathrm{H}(t-\varsigma(u) x),
$$

where $\mathrm{H}$ denotes the Heaviside distribution.

For this solution to be valid in the classical sense, that is $u \in X$, where $X$ is the function space for the classical solution, namely $X=C^{1}(\Omega)$, where $\Omega=\left\{(x, t) \in \mathbb{R}^{2} \mid 0<x<\ell, 0<\right.$ $t<\infty\}$, we will require $f \in C^{1}(0, \ell), h \in C^{1}[0, \infty)$, and $c \in C^{1}[X]$. For weak solutions to (2.1) these restrictions can be weakened, however we will require further regularity conditions on these functions when solving inverse problems. The regularity requirements used in the theoretical sections of this paper can be relaxed but as our purpose is not to provide the strongest possible results, but only to describe our methods, we use the most straight-forward regularity conditions.

For the formulation to make sense for an inverse problem, $u$ must be well defined and shocks must be avoided. When the problem is a pure initial-value one, John [8], page 16 has appropriate conditions on $h$ for $c(u)=u$, and on page 18 for $c(u)$. We extend that result for the problem (2.1).

Proposition 2.1. The solution to equation (2.1) is not singular, within the open rectangle $\Omega$, provided $f(0)=h(0)$, and if either of the following two conditions prevail:

A: $c$ increasing, or equivalently $\varsigma$ decreasing, while $f$ is strictly increasing and $h$ is strictly decreasing, within $\Omega$.

B: $c$ decreasing, or equivalently $\varsigma$ increasing, while $f$ is strictly decreasing and $h$ is strictly increasing, within $\Omega$.

Proof. Consider first the characteristic traces emanating from the line $t=0$, with $0 \leq x \leq$ $\ell$, on which the initial condition $u(x, 0)=f$ is specified. The characteristic traces can be written as $x=c(f(\tau))+\tau$, where $\tau$ is a parameter along the $x$-axis parameterising each 
trace. If two such traces intersect within $\Omega$ then the $t$ intercept value is

$$
\begin{aligned}
t & =-\frac{\left(\tau_{2}-\tau_{1}\right)}{c\left(f\left(\tau_{2}\right)\right)-c\left(f\left(\tau_{1}\right)\right)} \\
& =-\frac{1}{(c \circ f)^{\prime}(\tilde{\tau})}
\end{aligned}
$$

where the prime on $c$ denotes its derivative with respect to its argument, and the $\tau$ values distinguish the two traces, with $\widetilde{\tau}$ lying within the interval $\left[\tau_{1}, \tau_{2}\right]$. It is seen that a shock cannot occur for $t>0$ if $c \circ f$ is increasing.

Consideration of the characteristic traces emanating from the line $x=0$, and using a similar argument, shows that $\varsigma \circ h$ must be increasing.

To solve the inverse problem it will be necessary for $f$ and $h$ to have inverses. It follows that $f$ and $h$ must be strictly monotone which explains the slightly stronger than necessary conditions we have put on these functions.

Unless the consistency condition $f(0)=h(0)$ is met, a discontinuity and/or a fan will propagate from the point $(0,0)$.

The proposition now follows on noting that the wavespeed is the same function for both sets of characteristic traces.

It follows that if $f$ is compactly supported that shocks will occur; however these will not occur for $x<\ell$, and so will not concern us. The conditions may be relaxed to allow either $h \equiv 0$, or $f \equiv 0$, for the inverse problem without difficulties. For realistic physical models it is also assumed that $c \neq 0$, and henceforth we assume $c>0$.

2.1. Inverse Problem with measured transmission data. It is now possible to pose an inverse problem for reconstruction of the functional $c(\xi)$ from $(2.1)$, when the measured data is the time history of $u$ down-stream, that is $u(\ell, t)$. In order to do this $f$, and $h$ must be known functions. If $\bar{u}(t)=u(\ell, t)$ is the measured function, then an inverse reconstruction map is determined by the inverse of the map from the wavespeed $c$ to the measurement, namely through the implicit equation

$$
\bar{u}(t)= \begin{cases}f(\ell-c(\bar{u}) t), & 0<t<\ell / c(f(0)), \\ h(t-s(\bar{u}) \ell), & t>\ell / c(h(0)),\end{cases}
$$

which can easily be found from (2.2). If we define define the map, from the wavespeed $c$ to the measurement $\bar{u}$, by the operator $\mathbb{T}$, and assume that the measurement function and the wavespeed function required to be reconstructed are in appropriate function spaces represented by $J$ and $\Sigma$, respectively, then $\mathbb{T}: J \rightarrow \Sigma$. What is then required for the resolution of the inverse problem is an inverse operator map $\mathbb{T}^{-1}$. This can readily be found analytically as

$$
c(\bar{u})= \begin{cases}\left(\ell-f^{-1}(\bar{u}(t))\right) / t, & 0<t<\ell / c(f(0)) \\ \ell /\left(t-h^{-1}(\bar{u}(t))\right), & t>\ell / c(h(0)) .\end{cases}
$$


This solution may also be written as

$$
c(\xi)= \begin{cases}\left(\ell-f^{-1}(\xi)\right) / \bar{u}^{-1}(\xi), & 0<t<\ell / c\left(\xi_{0}\right), \\ \ell /\left(\bar{u}^{-1}(\xi)-h^{-1}(\xi)\right), & t>\ell / c\left(\xi_{0}\right),\end{cases}
$$

with $t=\bar{u}^{-1}(\xi)$ and if $f(0)=h(0)=\xi_{0}$. To use this form of solution it is required that $\bar{u}$ is strictly monotone; that this is the case follows from the next lemma. Prior to this let us define for future reference the time $t_{1}=\ell / c(f(0))=\ell / c(h(0))$, which is the time at which the characteristic from the point $(0,0)$ in the $(x, t)$-plane intersects the line $x=\ell$.

Note that this operator $\mathbb{T}$ is an implicitly defined map in the equation (2.3). For an extension of these ideas to problems where the inverse map cannot be defined analytically see [7].

Lemma 2.2. The function $\bar{u}$ is strictly monotonic, with the assumptions that the functions $f, h$ and $c$ are continuously differentiable, and with the condition $f(0)=h(0)$, and also either of the monotonicity conditions $A$ or $B$ being satisfied (see Proposition 2.1).

Proof. Upon differentiation of (2.3) with respect to $t$ it follows

$$
\bar{u}^{\prime}(t)= \begin{cases}-\frac{c(\bar{u}(t)) f^{\prime}(\ell-c(\bar{u}(t)) t)}{1+t c^{\prime}(\bar{u}(t)) f^{\prime}(\ell-c(\bar{u}(t)) t)}, & 0<t<\ell / c\left(\xi_{0}\right), \\ \frac{h^{\prime}(t-\varsigma(\bar{u}(t)) \ell)}{1+\ell \varsigma^{\prime}(\bar{u}(t)) h^{\prime}(t-\varsigma(\bar{u}(t)) \ell)}, & t>\ell / c\left(\xi_{0}\right),\end{cases}
$$

and if $f^{\prime}>0, h^{\prime}<0$ with $c^{\prime} \geq 0$ it follows $\bar{u}(t)$ is strictly decreasing. Similarly if $f^{\prime}<0$, $h^{\prime}>0$ with $c^{\prime} \leq 0$ it follows $\bar{u}(t)$ is strictly increasing.

From the solution (2.5) it is readily seen that $f$ and $h$ must be homeomorphisms and this follows from the earlier assumptions on these functions. It is also seen that the measurement function must also be homeomorphic, that this is the case follows from the open mapping theorem.

Corollary 2.3. The function $\bar{u}$ is a homeomorphism, with the assumptions of Lemma 2.2.

From the result (2.4), together with Lemma 2.2 it is seen that knowledge and control of $f$ or $h$ enable reconstruction of the functional $c$ to be made. Each of these functions reconstructs a part of $c$, and this can be more easily seen by consideration of the two simpler inverse problems:

- When $f$ is known, and $h \equiv 0$, it is possible to reconstruct $c(\xi)$ for the two cases as considered in the assumptions $\mathrm{A}$ and $\mathrm{B}$ from Proposition 2.1

with assumption $\mathrm{A}$ : As $\bar{u}$ is a strictly decreasing function, $\bar{u}\left(t_{1}\right) \leq \xi \leq \bar{u}(0)$, with $c$ an increasing function of $\xi$.

with assumption $\mathrm{B}$ : As $\bar{u}$ is a strictly increasing function, $\bar{u}(0) \leq \xi \leq \bar{u}\left(t_{1}\right)$, with $c$ a decreasing function of $\xi$.

- When $h$ is known, and $f \equiv 0$, it is possible to reconstruct $c(\xi)$ for the two cases as considered in the assumptions $\mathrm{A}$ and $\mathrm{B}$ from Proposition 2.1 
with assumption $\mathbf{A}: A s \bar{u}$ is a strictly decreasing function, $\bar{u}\left(t_{2}\right) \leq \xi \leq \bar{u}\left(t_{1}\right)$, with $c$ an increasing function of $\xi$.

with assumption $\mathrm{B}: \mathrm{As} \bar{u}$ is a strictly increasing function, $\bar{u}\left(t_{1}\right) \leq \xi \leq \bar{u}\left(t_{2}\right)$, with

$c$ a decreasing function of $\xi$.

In the aforementioned cases $t_{2}$ is the maximum time for which $\bar{u}$ has been measured.

If neither $f$ or $h$ are the zero function, and $f(0)=h(0)$, the first case in formula (2.4) is used until it is found that $t=\ell / c\left(\xi_{0}\right)$, then the second case in formula (2.4) is next utilised. The case $f(0) \neq h(0)$ can also be handled if it is known that both $f$ and $h$ are regular. This is because then the jump in $\bar{u}$ will determine $t_{1}$. The wavespeed $c$ will then be defined over an overlap region if $f(0)>h(0)$; otherwise $c$ will be undefined over a region.

It is seen that with the assumptions made, the solution of the inverse problem is unique and that the solution depends continuously upon the measured data $\bar{u}$, provided this data is continuous and is monotone.

Theorem 2.4. The wave speed functional $c(u)$ can be uniquely reconstructed, from transmission data $\bar{u}$, when $f$ and $h$ are appropriately chosen and $f(0)=h(0)$.

An important part of this result is that the inverse problem as posed here is wellconditioned. Although with real measurement data it is necessary to ensure existence of the inverse function of $\bar{u}$, this can be done by employing a monotone smoothing approximation to the sampled measured time function. Another aspect of the result is that wavespeed reconstruction is possible with purely transmission data, here particular reference is made to the reconstruction with knowledge of $h$. This contrasts sharply with the linear oneway wave equation, when it is desired to reconstruct $c(x, t)$ from transmission data from $h$ (see [10]). In the later case all that can be determined is the travel time through the medium. However it must be remarked although superficially similar, it is quite a different problem to the reconstruction of the wavespeed functional discussed here; as here $c$ is only dependent on $x$ and $t$ through the dependent variable $u$.

2.2. Inverse Problem with measured boundary flux data. An interesting adaptation of the problem would be to measure flux data at $x=0$, instead of the field at $x=\ell$. This follows the overspecified initial-boundary value technique used by [4] for a nonlinear wave problem, and [3] for a nonlinear diffusion equation; see also [5]. To determine $u_{x}(0, t)$ by measurement techniques will generally mean that $u$ be measured at two stations, such as $x=0$ and $x=x_{1}$, where $x_{1} \ll 1$, then $u_{x}(0, t)$ can be estimated by numerical differentiation techniques.

Manipulation of equation (2.1) when the side data

$$
u(0, t)=h(t), \quad u_{x}(0, t)=g(t), \quad 0 \leq t \leq \infty,
$$

are appropriate shows

$$
c(u(x, t))=-u_{x}(x, t) / u_{t}(x, t) .
$$

By then considering the limit of this equation as $x \rightarrow 0$ it follows

$$
c(h(t))=\lim _{x \rightarrow 0} c(u(x, t))=-g(t) / h^{\prime}(t) .
$$


If $h$ is strictly monotonic, then by writing $\xi=h(t)$ it follows

$$
c(\xi)=-g\left(h^{-1}(\xi)\right) / h^{\prime}\left(h^{-1}(\xi)\right) \text {. }
$$

Although this provides an elegant solution to the inverse problem, it is not such a practical solution as that of $\S 2.1$ due to, firstly the difficulty of measuring the boundary flux, and secondly, the resultant ill-conditioning of the problem. This ill-conditioning is because measurement of the flux at $x=0$ will be a differentiation operation.

\section{Semi-linear Source Problem}

Now consider the semi-linear one-way wave equation, with a source term $S$

$$
u_{t}+c(z) u_{z}=S(u), \quad 0<z<\ell, \quad t>0,
$$

for the dependent variable $u(z, t)$, as an initial-boundary value problem with the side conditions

$$
\begin{array}{ll}
u(0, t)=h(t), & 0 \leq t \leq \infty, \\
u(z, 0)=\bar{f}(z), & 0 \leq z \leq \ell .
\end{array}
$$

Here we assume the parameter $c$, is only a function of the spatial variable $z$. It is to be noted that the source term maybe considered as a reaction term which is a nonlinear function of $u$.

For this problem the characteristic traces can easily be straightened, via a transformation of the form

$$
x=\zeta(z)=\int_{0}^{z} \varsigma\left(x^{\prime}\right) d x^{\prime} .
$$

This transformation converts (3.1) into the form

$$
u_{t}+u_{x}=S(u), \quad 0<x<L, \quad t>0,
$$

where $L=\zeta(\ell)$. This equation for $u(x, t)$ has side conditions

$$
\begin{aligned}
u(0, t) & =h(t), \quad 0 \leq t \leq \infty, \\
u(x, 0) & =f(x) \equiv\left(\bar{f} \circ \zeta^{-1}\right)(x), \quad 0 \leq x \leq L,
\end{aligned}
$$

and the initial condition in the $x$-coordinates has been redefined by the $f$ function ${ }^{1}$. This formulation is more convenient for our subsequent development.

An implicit solution to equation (3.3) can be found to be

$$
\begin{cases}\int_{f(x-t)}^{u(x, t)} \frac{d \xi}{S(\xi)}=t, & 0<t<x \\ \int_{h(t-x)}^{u(x, t)} \frac{d \xi}{S(\xi)}=x, & t>x>0\end{cases}
$$

\footnotetext{
${ }^{1}$ Note $\zeta^{-1}$ exists as $\varsigma>0$
} 
where the solution is continuous across the line $t=x$ if $f(0)=h(0)$. By defining the functional

$$
T(\eta)=\int_{0}^{\eta} \frac{d \xi}{S(\xi)}
$$

this solution can be more conveniently described by

$$
\begin{cases}T(u(x, t))-T(f(x-t))=t, & 0<t<x \\ T(u(x, t))-T(h(t-x))=x, & t>x>0 .\end{cases}
$$

It can be easily verified that (3.5), or equivalently (3.4), is a classical solution to (3.3) with the given side conditions if $h \in C^{1}, f \in C^{1}$ and $S \neq 0$. Furthermore if $c>0$ and $c \in C^{1}$ then (3.5) is also a solution to (3.1).

3.1. Inverse Source Problem with measured transmission data. It is now possible to pose an inverse problem for reconstruction of the functional $S(u)$ from (3.1), when the measured data is the time history of $u$ down-stream, that is $u(\ell, t)$. In order to do this $\bar{f}, c$, and $h$, must be known functions. If $\bar{u}(t)=u(\ell, t)$ is the measured function then an inverse reconstruction map is easily determined by the inverse of the map from the nonlinear source function $S$ to the field. This is defined implicitly from

$$
\begin{cases}T(\bar{u}(t))-T(f(L-t))=t, & 0<t<L, \\ T(\bar{u}(t))-T(h(t-L))=L, & t>L .\end{cases}
$$

If we define this map by the operator $\mathbb{T}$, and assume that the measurement function $\bar{u}$ and the source function required to be reconstructed are in appropriate function spaces represented by $J$ and $\Sigma$, respectively, then $\mathbb{T}: J \rightarrow \Sigma$. What is required for the resolution of the inverse problem is an inverse operator map $\mathbb{T}^{-1}$. Equations (3.6) are functional equations the solution of which defines $\mathbb{T}^{-1}$ and yields $S$.

An explicit form of the map $\mathbb{T}$ can be found from (3.6) by use of the inverse of the functional $T$. The inverse will exist as $T$ is monotone, because $T^{\prime}(\eta)=1 / S(\eta)$ with either $S>0$ or $S<0$. By applying $T^{-1}$ to $(3.6)$ it is found

$$
\bar{u}(t)= \begin{cases}T^{-1}(t+T(f(L-t))), & 0<t<L, \\ T^{-1}(L+T(h(t-L))), & t>L .\end{cases}
$$

This shows the continuous dependence of the measurement function on the data $f$ and $h$.

3.2. Reconstruction of the source functional with only an applied boundary condition. As will be seen later, the initial function $f$ is not necessary for the reconstruction; so we first consider the problem where it is assumed that $f \equiv 0$. The operator $\mathbb{T}$ is 
then implicitly defined by

$$
\begin{cases}T(\bar{u}(t))=t & 0<t<L \\ T(\bar{u}(t))-T(h(t-L))=L, & t>L\end{cases}
$$

and with the assumption that $h$ is continuously differentiable an explicit formula maybe found for $S(\xi)$. By differentiation of the formulae in (3.7) with respect to $t$, and after some manipulation, the source functional may be written as

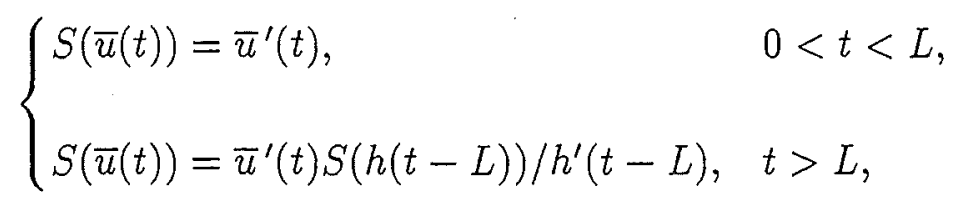

where use has been made of the fact that $T^{\prime}(\xi)=1 / S(\xi)$. Now with the assumption that the boundary value function $h$ is strictly monotone and continuous, we may define $t=\bar{u}^{-1}(\xi)$. It will be shown in Corollary 3.2 that the monotonicity assumption on $h$, and suitable conditions on $S$, ensures that $\bar{u}$ is a homeomorphism; hence $\bar{u}^{-1}$ exists. Now (3.8) may be rewritten as

$$
\begin{cases}S(\xi)=\bar{u}^{\prime}\left(\bar{u}^{-1}(\xi)\right), & \bar{u}(0)<\xi<\bar{u}(L), \\ S(\xi)=\bar{u}^{\prime}\left(\bar{u}^{-1}(\xi)\right) S\left(h\left(\bar{u}^{-1}(\xi)-L\right)\right) / h^{\prime}\left(\bar{u}^{-1}(\xi)-L\right), & \bar{u}(L)<\xi\end{cases}
$$

We see that the resolution of this inverse problem is ill-posed, and this follows as it requires a differentiation operation on the measurement function. However if $\bar{u}$ is mollified (see [10] and [9]), it will follow that the resultant regularised problem depends continuously on the measurement data and so is well-posed.

Lemma 3.1. The function $\bar{u}$ is strictly monotonic, with the assumption that either $S>0$ and $h$ strictly increasing, or $S<0$ and $h$ strictly decreasing.

Proof. First consider the case that $0<t<L$, then the first formula in (3.8) shows if $S>0$ we have $\bar{u}^{\prime}>0$, and if $S<0$ then $\bar{u}^{\prime}<0$, the result follows immediately. For $t>L$ the second formula in (3.8) can be manipulated into the form

$$
\bar{u}^{\prime}(t)=S(\bar{u}(t)) h^{\prime}(t-L) / S(h(t-L)) .
$$

From this equation if $S>0$ and $h^{\prime}>0$ then $\bar{u}^{\prime}>0$, and conversely if $S<0$ and $h^{\prime}<0$ then $\bar{u}^{\prime}<0$. The strictly monotonicity result follows.

It is also seen that the measurement function must also be homeomorphic, that this is the case follows from the open mapping theorem.

Corollary 3.2. The function $\bar{u}$ is a homeomorphism, with the assumptions of Lemma 3.1. 
It remains to show how the equation (3.9) can be used to recursively reconstruct the functional form of $S$. The first part is trivial. To solve the second part of this functional equation the range of the function $h$ (and hence of $\bar{u}$ ) will determine how much of $S$ can be reconstructed.

It is readily seen that from equation (3.4) that when $t=0$

$$
\int_{0}^{\bar{u}(t)} \frac{d \xi}{S(\xi)}=0
$$

and this implies that $\bar{u}(0)=0$, as $S \neq 0$. We shall now for illustrative purposes assume that $h(0)=0$ and $h(t) \rightarrow \infty$ as $t \rightarrow \infty$. Hence provided $\bar{u} \in C^{1}$, the first part of (3.9) provides a functional solution for $S(\xi)$, with $\xi \in[0, \bar{u}(L)]$. Now with $\xi \in\left[\bar{u}(L), \bar{u}\left(L+h^{-1}(\bar{u}(L))\right]\right.$ it is seen that the argument of the term $S\left(h\left(\bar{u}^{-1}(\xi)-L\right)\right.$ ), on the right-hand-side of the second part of (3.4), is in the range $[0, \bar{u}(L)]$. Hence it is now possible to reconstruct $S(\xi)$, with $\xi \in\left[\bar{u}(L), \bar{u}\left(L+h^{-1}(\bar{u}(L))\right]\right.$. Then for $\xi>\bar{u}\left(L+h^{-1}(\bar{u}(L))\right.$ we may continue to reconstruct $S(\xi)$ in a similar manner. It is seen that $S$ can therefore be completely reconstructed from the inverse map equations (3.9).

Theorem 3.3. The source functional $S(u)$ can be uniquely reconstructed, from transmission data $\bar{u}$, with an appropriately chosen boundary function $h$.

3.3. Reconstruction of the source function with only initial conditions. If $h \equiv 0$ and $f \neq 0$ then the operator $\mathbb{T}$ is defined by

$$
\begin{cases}T(\bar{u}(t))-T(f(L-t))=t, & 0<t<L, \\ T(\bar{u}(t))=L, & t>L,\end{cases}
$$

By differentiation of the formulae in (3.10) with respect to $t$, and after some manipulation, the source functional may be written as

$$
S(\bar{u}(t))=\bar{u}^{\prime}(t) S(f(L-t)) /\left(S(f(L-t))-f^{\prime}(t-L)\right), \quad 0<t<L .
$$

For this case $\bar{u}^{\prime}(t)=0$, when $t>L$, as the intial condition function has completely propagated past the point $x=L$ after a time $t=L$. Therefore the only information on $S$ is obtained from the measurement for $0<t<L$. The functional equation (3.11) does not admit an explicit solution. We shall not pursue this problem any further.

3.4. Inverse source problem with measured boundary flux data. An adaptation of the approach of $\S 2.2$ would be to measure flux data at $z=0$, instead of the field at $z=\ell$. This allows for the overspecified initial-boundary value technique used earlier. To determine $u_{z}(0, t)$ will generally mean that $u$ be measured at two stations, such as $\dot{z}=0$ and $z=z_{1}$, where $z_{1} \ll 1$, then $u_{z}(0, t)$ can be estimated by numerical differentiation techniques.

Manipulation of (3.1) when the side data

$$
u(0, t)=h(t), \quad u_{z}(0, t)=g(t), \quad 0 \leq t \leq \infty,
$$


are appropriate shows

$$
S(u(z, t))=u_{t}(x, t)+c(z) u_{z}(z, t)
$$

and on considering the limit of this equation as $z \rightarrow 0$, it follows

$$
S(h(t))=\lim _{x \rightarrow 0} S(u(z, t))=h^{\prime}(t)+g(t) c(0) .
$$

If $h$ is strictly monotonic, then writing $\xi=h(t)$ enables this equation to be represented as

$$
\left.S(\xi)=h^{\prime}\left(h^{-1}(\xi)\right)\right)+g\left(h^{-1}(\xi)\right) c(0) \text {. }
$$

Although this again provides an elegant solution to the inverse problem, it is not so practical as the solution of $\S 2.1$ due to the difficulty of measuring the boundary flux, and also, the resultant ill-conditioning of the problem.

\section{SUMMARY}

Explicit functional solutions have been found for several inverse problems associated with nonlinear one-way wave equations. For the functional wavespeed reconstruction problem the solution is well-posed. This is not the case when the inverse problem is one of functional reconstruction of the source term. These problems as considered, are simple, but they are special cases of more complex problems, whose solutions will have similar characteristics to those presented here.

The two theorems presented in this paper giving global uniqueness results for our reconstruction methods are of interest, this is because for many inverse problems only a local uniqueness result is available.

The results presented in this paper illustrate the viability of the application of current methods, of solution for inverse problems, associated with linear time-dependent problems to those associated with nonlinear time-dependent problems. Of course for more complex nonlinear equations than those considered here, one cannot expect to find analytic solutions to a particular inverse problem. Then recursion and linearisation techniques must be employed, we shall consider such problems in a later paper.

\section{ACKNOWLEDGEMENTS}

One of the authors (T.J.C) wishes to express his gratitude to the Department of Mathematics and Statistics at the University of Canterbury for a grant enabling this research to be performed.

\section{REFERENCES}

[1] Ingegerd Åberg, Gerhard Kristensson, and David J.N. Wall. Transient waves in non-stationary media. Technical Report LUNDEDX/(TEAT-7037)/1-23/(1994), Teoretisk Elektroteknik, Tekniska Högskolan i Lund. To appear J. Math. Phys. 1996.

[2] Ingegerd Åberg, Gerhard Kristensson, and David J.N. Wall. Propagation of transient electromagnetic waves in time-varying media-direct and inverse scattering problems. Inverse Problems, 11(1):29-49, 19.95.

[3] J.R. Cannon and P. DuChateau. An inverse problem for an unknown source term in a heat equation. J. Math. Anal. Appl., 7S(1):465-485, 1980. 
[4] J.R. Cannon and P. DuChateau. An inverse problem for an unknown source term in a wave equation. SIAM J Appl. Math., 43(3):553-564, 1983.

[5] J.R. Cannon and H-M. Yin. On a class of nonlinear parabolic equations with nonlinear trace functionals. Inverse Problems, 7(1):149-162. 1991.

[6] T.J. Connolly. Non-linear Methods for Inverse Problems. PhD thesis, University of Canterbury, Christchurch, New Zealand, 1990.

[7] T.J. Connolly and D.J.N. Wall. On Fréchet differentiability of some non-linear operators occurring in inverse problems; an implicit function theorem approach. Inverse Problems, 6:949-966, 1990.

[8] F. John. Partial differential equations. Springer Verlag, New York Heidelberg Berlin, third edition, 1978.

[9] Diego A Murio. The Mollification Method and the Numerical Solution of Ill-Posed Problems. John Wiley \& Sons, Inc, New York, N:Y., 1993.

[10] David J.N. Wall and Jonas Lundstedt. Inverse source problems involving the one-way wave equation: Source reconstruction. Technical Report LUNDEDX/(TEAT-?)/1-25/(1996), Teoretisk Elektroteknik, Tekniska Högskolan i Lund, Lund, Sweden, 1996.

Department of Mathematics \& Statistics, University of Canterbury, Christchurch, 1 , NEW ZEALAND

Department of Mathematics \& Statistics, University of Canterbury, Christchurch, 1 , NEW ZEALAND

E-mail address: D. Wall@math. canterbury,ac.nz 